\title{
Random vs. Deterministic Deployment of Sensors in the Presence of Failures and Placement Errors
}

\author{
Paul Balister \\ University of Memphis \\ pbalistr@memphis.edu
}

\author{
Santosh Kumar \\ University of Memphis \\ santosh.kumar@memphis.edu
}

\begin{abstract}
Although random deployment is widely used in theoretical analysis of coverage and connectivity, and evaluation of various algorithms (e.g., sleep-wakeup), it has often been considered too expensive as compared to optimal deterministic deployment patterns when deploying sensors in real-life. Roughly speaking, a factor of $\log n$ additional sensors are needed in random deployment as compared to optimal deterministic deployment if $n$ sensors are needed in a random deployment. This may be an illusion however, since all real-life large-scale deployments strategies result in some randomness, two prime sources being placement errors and sensor failures, either at the time of deployment or afterwards.

In this paper, we consider the effects of placement errors and random failures on the density needed to achieve full coverage when sensors are deployed randomly versus deterministically. We compare three popular strategies for deployment. In the first strategy, sensors are deployed in an optimal lattice but enough sensors are colocated at each lattice point to compensate for failure and placement errors. In the second, only one sensor is deployed at each lattice point but lattice spacing is sufficiently shrunk to achieve a desired quality of coverage in the presence of failure and placement errors. In the third, a random deployment is used with appropriate density.

We derive explicit expressions for the density needed for each of the three strategies to achieve a given quality of coverage, which are of independent interest. In comparing the three deployments, we find that if errors in placement are half the sensing range and failure probability is $\mathbf{5 0 \%}$, random deployment needs only around $10 \%$ higher density to provide a similar quality of coverage as the other two. We provide a comprehensive comparison to help a practitioner decide the lowest cost deployment strategy in real-life.
\end{abstract}

\section{INTRODUCTION}

Given the ever increasing number of new applications that are becoming possible with wireless sensors, the importance of educated deployments of wireless sensors continues to increase. Knowing the appropriate number of sensors to deploy is critical to ensuring that a desired quality of monitoring is achieved at the lowest possible cost. Although optimal patterns of deployment to ensure full coverage (i.e., covering every point in the region) when deploying sensors deterministically, and critical density needed to achieve full coverage when deploying randomly, has been studied extensively [2], [7], [9][11], joint effects of placement errors and sensor failures on the optimal/critical densities has not been addressed before.

It is known that triangular lattice is an optimal pattern of deployment to achieve full coverage [2]. It is also known that if sensors are placed accurately and they are $100 \%$ reliable, optimal deterministic deployment will need $O(\log n)$ times fewer sensors versus a random deployment, where $n$ is the number of sensors needed in a random deployment [7]. Thus it appears that deterministic deployments are far superior. However, such a conclusion is illusory. For one, placing sensors in such a pattern is cumbersome, at best. Second, placement errors are inherent, and they introduce coverage holes in the region. Third, failure of sensors, which is also quite likely (especially in an outdoor unattended deployment [5]), will cause even larger coverage holes.

One intuitive method to address both of these perturbations is to place multiple sensors at each point of the triangular lattice $(\mathrm{OPT})$. Another approach is to place sensors in a lattice (or grid), but scale down the grid spacing sufficiently so as to achieve full coverage with this fine lattice (FLAT). This model was first studied in [8] for unreliable sensors and subsequently refined in [7], [11]. Placement errors are, however, not considered in either work.

The third approach, which is widely used in theoretical studies and simulations, is to deploy sensors randomly according to a uniform Poisson process (RAND). In this case, placement error has no effect, since a Poisson deployment with placement error is still a Poisson deployment. Similarly, Poisson deployment with random failures is again a Poisson deployment but with lower density. The density needed to achieve full coverage for random deployment has been extensively studied [7], [9], [10]. However, these give only asymptotic results, and it is not clear how large $n$ must be for the estimates to become sufficiently accurate, limiting their usefulness in practice.

In this paper, we investigate the three deployment strategies described in the preceding for their relative efficiency. To make our results more useful for practitioners, we use the number of coverage holes as our metric. Traditionally, area of vacant region has been used to indicate the extent of coverage [9], [10]. Notice that even if the area of vacant region is small, there could be many coverage holes. On the other hand, if the number of coverage holes $<1$, it implies full coverage.

We make the following contributions in this paper:

1) Comprehensive treatment of one dimension: We give reliable, explicit estimates of the density needed in each of the three deployment strategies for given failure probability and given degree of placement error in the 1-dimensional case. (Section III) 
2) Reliable density estimates in two dimensions: We give reliable estimate of density needed for RAND, but for the other two strategies only when the placement error is zero. For FLAT and OPT with non-zero placement errors, the analysis becomes highly involved. Our estimates for RAND are much more accurate than known asymptotic critical conditions, making them useful for practical deployments. Previously, only asymptotic critical conditions for the FLAT deployment under failures were known [7], [11]. For OP T, we are not aware of any existing work that considers sensor failures. (Section IV)

3) Guidance on when to use which: We compare each of the three deployments for various values of failure probabilities and placement errors and point out the difference in density therein.

\section{Model And Key Observations}

Consider a wireless sensor network deployed in a large area. For simplicity we shall assume all sensors detect events within a disk, with common sensing range $r=1$. (Similar results will in fact hold for most plausible shapes of sensing region.) We model the error in sensor placements by a symmetric bivariate gaussian distribution with probability density function $\frac{1}{2 \pi \sigma^{2}} \exp \left(-\left\|x-x_{0}\right\|^{2} / 2 \sigma^{2}\right)$, where $x \in \mathbb{R}^{2}$ is the actual sensor position, $x_{0} \in \mathbb{R}^{2}$ is the target position, and $\sigma$ is the standard deviation in each coordinate of the vector $x-x_{0}$. We shall also assume sensors are active with probability $p$ (i.e., fail with probability $1-p$ ). Finally, we assume the errors in location and failure events of each sensor are independent, and independent of all the other sensors. Note that failure independence has been observed in real-life deployments [5].

If we wish to minimize the number of sensors required, then triangular grid placement is known to be optimal with an asymptotic density of $2 / \sqrt{27} \approx 0.3849$ [2]. Note the obvious lower bound of $1 / \pi \approx 0.3183$, since each sensor can only sense a region of area $\pi$. It is also known that for random uniform deployment, the density of sensors required is asymptotically about $\frac{1}{\pi}(1+o(1)) \log |\mathcal{A}|$ where $|\mathcal{A}|$ is the area of the deployment region $\mathcal{A}$. Since this tends to infinity as $|\mathcal{A}| \rightarrow \infty$, it seems that deterministic deployment is far superior to random deployment. This would indeed hold if there were no failures or placement errors. However, for any constant value of $p<1$ the density of sensors needed to ensure a reasonable chance of coverage will be roughly proportional to $\log |\mathcal{A}|$. Indeed, if we divide the region $\mathcal{A}$ into about $|\mathcal{A}| / 9$ squares of side length 3 , and if each of these squares has at most $\varepsilon \log |\mathcal{A}|$ sensors, then we would expect that about $(1 / 9)|\mathcal{A}|(1-p)^{\varepsilon \log |\mathcal{A}|}=(1 / 9)|\mathcal{A}|^{1+\varepsilon \log (1-p)}$ of these squares not to contain any active sensor. Hence if $\varepsilon$ is small enough, one would expect many empty squares, and hence many coverage holes. Similarly, for any constant $\sigma>0$, the density of sensors needed will tend to infinity as $|\mathcal{A}| \rightarrow \infty$ (possibly more slowly than $\log |\mathcal{A}|$ ). Thus for full coverage of a large area we always need a large density in practice.

In addition to the three strategies introduced in Section I, namely OPT, FLAT, and RAND, we shall also consider strategies that are intermediate between OPT and FLAT, namely

FLAT $(k)$ : Deploy $k$ sensors at each lattice point (for some $k \geq 1$ ), but scale the lattice as in FLAT.

Note that FLAT (1) is the same as FLAT. For simplicity, and to avoid dependency on the area $|\mathcal{A}|$ of the deployment region, we shall consider deployment in the infinite plane. Reliability will be assessed by considering the asymptotic density of coverage holes, i.e., the limit

$$
I=\lim _{|\mathcal{A}| \rightarrow \infty} \frac{N_{\mathcal{A}}}{|\mathcal{A}|},
$$

where $N_{\mathcal{A}}$ the expected number of uncovered regions in a disk $\mathcal{A}$, say, of area $|\mathcal{A}|$. It can be proved (using the SteinChen method, see [1]) that with any of the above strategies the number of coverage holes is approximately Poisson distributed with mean $I|\mathcal{A}|$, and this approximation improves for fixed $I|\mathcal{A}|$ as $I \rightarrow 0$ and $|\mathcal{A}| \rightarrow \infty$. Thus the probability of full coverage is well approximated by $e^{-I|\mathcal{A}|}$ for small $I$.

For illustration, we shall compare the hole densities $I$ of the different strategies for two dimensions. Figure 1 shows diagrams giving the hole densities using strategies $\mathrm{OPT}$, FLAT (1), FLAT (2), and FLAT (3) when the density of sensors used is sufficient to give strategy RAND a failure rate of $I=10^{-2}$, i.e., one coverage hole on average per $10^{2}$ units of area. Contour lines indicate by what factor the density of sensors needs to be increased when using strategy RAND so as to match the given strategy. This slightly strange representation was chosen since it allows simple comparison between the strategies, while avoiding the problem that the coverage hole densities of strategies OPT and FLAT $(k)$ can be rather erratic functions of the parameters when $\sigma$ is small. (The coverage hole density function $I$ for strategy RAND is well behaved see below.) In each case, we vary the deployment position error $\sigma$ and sensor reliability rate $p$. Figure 1 also gives a diagram for strategy $\mathrm{OPT}$ when $I=10^{-4}$, and a diagram indicating which strategy is best (for $I=10^{-2}$ ). We now make some key observations from these results.

Observation 1. The density of sensors required to achieve a given level of reliability does not vary much between the different strategies unless $p \approx 1$ and $\sigma \approx 0$.

The differences between the strategies decreases as $p \rightarrow 0$ (unreliable sensors) or $\sigma \rightarrow \infty$ (large errors in deployment) as one might expect. However, the sensor density for uniform deployment is only a few 10's of percent more throughout much of these diagrams. The differences between strategies OPT and FLAT $(k)$ are even smaller in general.

Observation 2. The ratio between the sensor densities required for each strategy is largely independent of $I$. For example, there is very little difference in the diagrams for $I=10^{-2}$ and $I=10^{-4}$.

Observation 3. The best strategies seem to be $\mathrm{OPT}$ (for low $p$ and low $\sigma$, or FLAT for large $\sigma$ or large $p$.

There are a few locations with small $\sigma$ where one of the other FLAT $(k)$ strategies wins, but mostly the choice is between OPT and FLAT. 


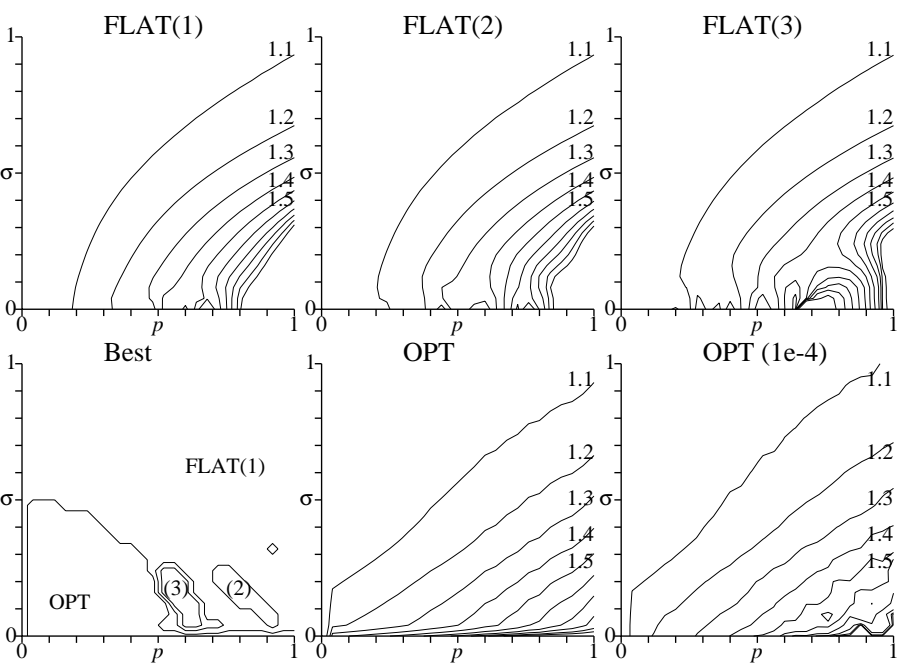

Fig. 1. Two-dimensional deployment. Ratio of densities needed as a function of $p$ and $\sigma$ for a fixed choice of $I_{\text {RAND }}\left(=10^{-4}\right.$ for bottom right and $10^{-2}$ for the rest). Contours show by what factor density must be increased in RAND to match reliability of strategy OPT or FLAT $(k)$. Also shown is a diagram (lower left) giving the best choice of these algorithms for different values of $p$ and $\sigma$.

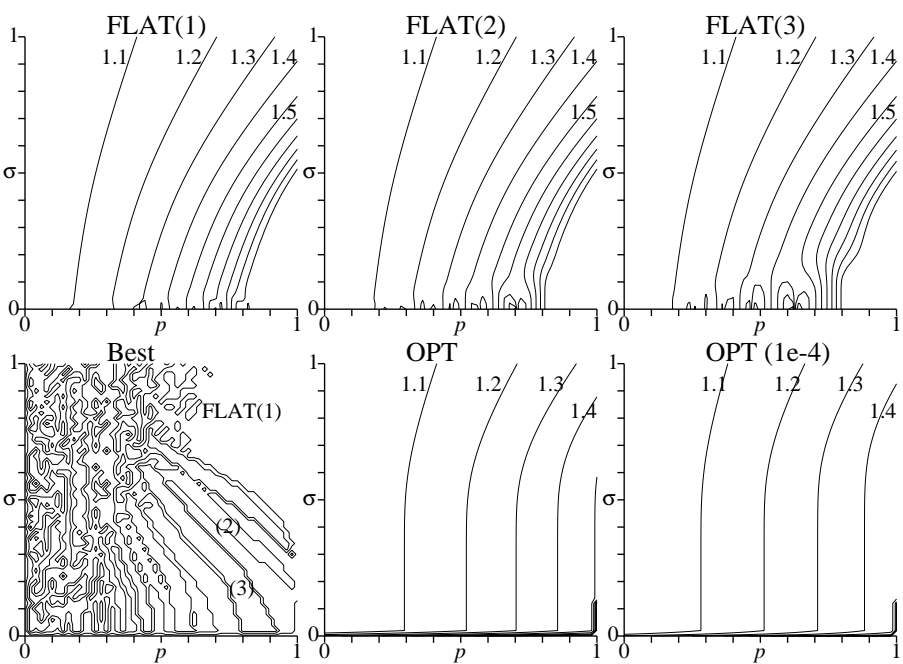

Fig. 2. One-dimensional deployment. The setup and legends are same as in Figure 1.

In the following sections we derive estimates for the coverage hole density which explain Observations 1 and 2, but also show that Observation 3 does not tell the whole story. In fact, as $I \rightarrow 0$, strategy FLAT always wins, while OPT is eventually worse than even the RAND strategy for small values of $p$.

One dimensional deployment. We also consider the case of one dimensional deployment where sensors are deployed along a line. Simulation results for this case are shown in Figure 2, which uses a similar framework as for the two dimensional case. There are a number of similarities between the results for one and two dimensional cases, however, there are also some very significant differences.

Observation 4. Strategy OPT performs badly in the onedimensional case when $\sigma>0$, and there is a discontinuity in performance at $\sigma=0$, while in the two dimensional case OP T can often be quite good, and there is no such discontinuity.

As we stated above, strategy OPT is only really good in the two dimensional case when $I$ is not too small. In the one dimensional case it is always bad for $\sigma>0$.

Observation 5. Strategy FLAT is usually beaten by some FLAT $(k), k>1$, in the one-dimensional case, whereas this is seldom true in the two dimensional case.

In the following sections, we provide analytic explanations for each of these observations.

\section{ONE-DIMENSIONAL DEPLOYMENTS}

We first present our results for the one dimensional case. We aim to study the number of sensor gaps in a long line where, for each $i \in \mathbb{Z}$, we place $k$ sensors with a normal distribution $N\left(i h, \sigma^{2}\right)$, and each sensor has a probability $p$ of being active. Here, $h$ is the lattice scale, whose value is decided by the particular deployment strategy. As discussed in Section II, we compare only the coverage hole densities since it suffices for comparing the probabilities of full coverage.

To count the expected number of coverage holes, consider the probability $p(x)$ that an active sensor (assumed to be at position $x$ ) is immediately followed by a gap of length 2 which does not contain another active sensor. When $\sigma>0$ one obtains (see [3] for more details)

$$
p(x)=\prod_{i=-\infty}^{\infty}\left(1-p \Phi\left(\frac{2+x-i h}{\sigma}\right)+p \Phi\left(\frac{x-i h}{\sigma}\right)\right)^{k-\delta_{i 0}},
$$

where $\phi(x)=\frac{1}{\sqrt{2 \pi}} e^{-x^{2} / 2}$ is the probability density function, and $\Phi(x)=\int_{-\infty}^{x} \phi(t) d t$ the cumulative function, of a $N(0,1)$ random variable, and $\delta_{i 0}=1$ when $i=0$ and 0 when $i \neq 0$. Multiplying by the density of sensors at $x$ and integrating over $x$ gives the density of coverage holes per unit length as

$$
I(k, h, p, \sigma)=\frac{k p}{h} \int_{-\infty}^{\infty} p(x) \phi\left(\frac{x}{\sigma}\right) \frac{1}{\sigma} d x .
$$

Although no closed form expression for $I(k, h, p, \sigma)$ seems likely, it can be evaluated numerically quite easily. For each of the strategies we fix the density of sensors to be $\rho$ sensors per unit length. Thus we require that $\rho=k / h$. For strategy OPT, we fix $h=2$ (so $k=2 \rho$ ) since it is clear that the optimal deployment involves placing sensors at intervals 2 apart. For strategy FLAT $(k)$, we fix $k$ (so $h=k / \rho$ ). For strategy RAND $p(x)=e^{-2 p \rho}$. (The number of active sensors in $[x, x+2]$ is Poisson with mean $2 p \rho$ for the uniform deployment.) Thus the intensity of coverage gaps is given explicitly by the formula

$$
I_{\mathrm{RAND}}=p \rho e^{-2 p \rho} \text {. }
$$

As $\sigma \rightarrow \infty$, all the above strategies converge to strategy RAND since the distribution of sensors becomes more uniform. To explain the differences between the strategies, it therefore helps to consider the case when $\sigma$ is small, in particular we shall consider the case $\sigma=0$, when there are no errors in positioning the sensors.

Fix $k, h$ and $p$ as above and $\sigma=0$. To obtain a coverage hole in the above model, we need at least one of $k$ sensors at 
one point to be active, but all of the next $\lfloor 2 / h\rfloor$ groups of $k$ sensors to fail. This occurs with probability $\left(1-(1-p)^{k}\right)(1-$ $p)^{k\lfloor 2 / h\rfloor}$, and can happen at each sensor location. Hence the density of holes is

$$
\begin{aligned}
I(k, h, p, 0) & =\frac{1}{h}\left(1-(1-p)^{k}\right)(1-p)^{k\lfloor 2 / h\rfloor} \\
& \approx \frac{\left(1-(1-p)^{k}\right)}{p k} p \rho(1-p)^{2 \rho},
\end{aligned}
$$

where the approximation is exact if $2 / h$ is an integer.

Fixing $\rho$ and letting $k$ vary, we see that the approximation to $I(k, h, p, 0)$ above decreases with increasing $k$. In particular, comparing strategy FLAT with FLAT $(k)$ we have $I_{\mathrm{FLAT}(\mathrm{k})} / I_{\mathrm{FLAT}}=\left(1-(1-p)^{k}\right) /(p k)$ which is always less than 1 . Of course this relies on $2 / h$ being an integer, so in practice $I_{\mathrm{FLAT}(\mathrm{k})}$ will fluctuate somewhat with $k$. We see in Figure 2 that the optimal value of $k$ varies erratically with the parameters. This is due to the rapid variations in the fractional part of the number $2 / h$.

Increasing $k$ however usually improves the strategy, and for the largest possible $k(h=2)$ we obtain strategy OPT. The reason is essentially that in all cases we need $k\lfloor 2 / h\rfloor \approx 2 \rho$ sensors to fail for a coverage hole to form, but for large $k$, there are fewer choices for these sensors that actually results in a hole. Now (3) and (4) can be inverted to find the approximate density $p \rho$ of active sensors needed when $|\mathcal{A}|$ is large:

$$
\begin{array}{ll}
\text { OPT } & p \rho \approx \frac{-p}{\log (1-p)} \log (2|\mathcal{A}|) \quad(\sigma=0) \\
\text { FLAT } & p \rho \approx \frac{-p}{\log (1-p)}(\log (2|\mathcal{A}|)+\log \log (2|\mathcal{A}|)) \\
\text { RAND } & p \rho \approx 1 .(\log (2|\mathcal{A}|)+\log \log (2|\mathcal{A}|))
\end{array}
$$

Note that $-p / \log (1-p)=1-\frac{p}{2}+O\left(p^{2}\right)$ is close to 1 when $p$ is small, and there is little difference between FLAT and OPT for any $p$.

For $\sigma>0$ but small, strategies FLAT $(k)$ are not usually significantly affected. However, strategy $\mathrm{OPT}$ is. The reason for this is that it is possible for each group of $k$ sensors to contain an active sensor, yet a small coverage hole forms due to the fact that the gap between the two groups is slightly more than 2. Suppose $\sigma$ is positive, but very small, so that to estimate the probability of a coverage hole between, say, $x=0$ and $x=2$, we can ignore the sensors which were targeted at points other than $x=0$ and $x=2$. Then one can show (see [3] and below) that for $\sigma>0$

$$
\text { OPT } \quad p \rho \approx \frac{-p / 2}{\log (1-p / 2)}\left(\log (2|\mathcal{A}|)+\frac{1}{2} \log \log (2|\mathcal{A}|)\right)
$$

which lies between strategies RAND and FLAT. Note that this is almost independent of $\sigma>0$, unless $\sigma$ is large enough that the sensor location error has a reasonable chance of being more than 2 units. We can see this on Figure 2, where the contour lines jump from $\sigma=0$ to $\sigma>0$, but then stay almost vertical until $\sigma$ is large. As discussed in Section II, Figure 2 shows how many additional sensors are needed in RAND to match the reliability of strategy FLAT $(k)$ and OPT.

For small $\sigma$ the best strategy is likely to be a slight modification of strategy OPT, where we scale the deployment pattern so that adjacent groups of sensors are just slightly less than 2 units apart. Effectively this is strategy $\operatorname{FLAT}(k)$ with a large, but carefully chosen $k$. For larger $\sigma$, differences between the different strategies become negligible.

\section{Two Dimensional DEPLOYMENTS}

For the 1-dimensional case, (2) (or (3) or (4)) allow adequate determination of the coverage hole intensity. For the 2-dimensional case, no such simple expression seems likely, so in general we will need to resort to numerical simulations. However, we have obtained the following result for the number of coverage holes in the case of uniform deployment.

Theorem 4.1: The coverage hole density for the uniform random deployment case in 2 dimensions is given by

$$
I_{\mathrm{RAND}}=p \rho(\pi p \rho-1) e^{-\pi p \rho}+p \rho(1+o(1)) e^{-4 \pi p \rho} .
$$

Due to space limitations, we defer the proof of Theorem 4.1 to [3]. Although there are estimates in the literature for the probability of full coverage, and for the number of coverage holes, the above estimate is far more accurate and the error terms can be explicitly bounded (see [3]), so that they can be applied in situations far from the " $n \rightarrow \infty$ " limit in which previous estimates have been derived. For situations in which one has a reasonable chance of full coverage, the second term above is in practice extremely small. As discussed in Section II, once hole intensity is known, probability of full coverage is given approximately by $e^{-I|\mathcal{A}|}$ (once again, the errors can be explicitly bounded), and hence a reliable estimate for density can be derived for any set of parameters.

Looking at Figure 1 we once again see that the strategies converge when $\sigma$ is large. To understand the difference in strategies for small $\sigma$ we again look first at the $\sigma=0$ case. First, let us consider strategy $\mathrm{OPT}$ with sensor density $\rho$.

Theorem 4.2: The coverage hole density for strategy $\mathrm{OPT}$ when $\sigma=0$ is given by

$$
I_{\mathrm{OPT}}=(2 / \sqrt{27})(1-p)^{k}+O\left((1-p)^{2 k}\right)
$$

where $k=\rho \sqrt{27} / 2$ is the number of sensors at each lattice point.

Proof: For a coverage hole to exist, an entire group of $k$ sensors must be inactive. For a fixed group this occurs with probability $(1-p)^{k}$. The number of coverage holes is at most the number inactive groups. On the other hand, if an inactive group is surrounded by six active groups then a hole is definitely formed. Thus the number of holes per lattice point is at least $(1-p)^{k}\left(1-(1-p)^{k}\right)^{6}=(1-p)^{k}+O\left((1-p)^{2 k}\right)$. The result follows as the area per lattice point is $\sqrt{27} / 2$.

For small $\sigma>0$ there does not seem to be a jump in reliability as there was in the one dimensional case. The derivation of $I_{\mathrm{OPT}}$ in the $\sigma>0$ case is much more involved than in the one dimensional case, but a simple heuristic offers some explanation. In the one dimensional case, a small coverage hole appears between lattice points with probability about $(1-p / 2)^{2 k} \sqrt{\pi k}$ (see [3]) which is more than the probability $(1-p)^{k}$ that an entire group of sensors are inactive. Ignoring the $\sqrt{\pi k}$ factor, $(1-p / 2)^{2 k}$ is just the probability that the mid-point between two sensor groups is uncovered. Indeed, there are $2 k$ sensors that could cover it, and each 
sensor covers it with probability $p / 2$ ( $p$ to be active, and $1 / 2$ due to positioning error). In the case of two dimensions, the mid-point of a lattice triangle is at distance $r$ from three lattice points, but is still covered by active sensors with probability $1 / 2$. Thus we have a probability of $(1-p / 2)^{3 k}$ that this mid-point is uncovered. It turns out that the probability of a small coverage hole near the center of a lattice triangle is about $(1-p / 2)^{3 k}$ times some small order term (power of $k$ ). However, for $p<.7639,(1-p / 2)^{3 k}$ is less than $(1-p)^{k}$, which is the probability that a hole appears due to an entire group of sensors being inactive. Thus there is generally not so much difference between the small $\sigma>0$ and $\sigma=0$ cases in two dimensions.

Finally, consider strategy FLAT (or FLAT $(k)$ ) in the case when $\sigma$ is small (or zero). Assuming the sensor density $\rho$ is large, the sensors are deployed on a very fine grid with spacing much less than $r$. One can follow the arguments used to prove Theorem 4.1 to derive an estimate for the coverage hole density.

Theorem 4.3: The coverage hole density for strategy FLAT (or FLAT ( $k$ ) for fixed $k$ ) is given for $\sigma \ll 1, \rho \gg 1$, by

$$
I_{\mathrm{FLAT}}=p \rho(\pi p \rho-1)(1-p)^{\pi \rho+O\left(\rho^{1 / 2}\right)} .
$$

The main difference is that the probability of a given point being uncovered (i.e., no active sensors within distance $r$ ) is $e^{-\pi p \rho}$ in the case of RAND, but $(1-p)^{\pi \rho+O\left(\rho^{1 / 2}\right)}$ in the case of FLAT. (The error term due both to the discreteness of the lattice, and also due to the placement error.) Also, the error in the exponent dominates the second term that one would expect by comparison with Theorem 4.1. Also, the only difference between FLAT and FLAT $(k)$ is that the constant implicit in the error term increases with increasing $k$ due to larger boundary effects when the grid is coarser. Similarly the error term increases with $\sigma$.

As in the one dimensional case we can invert the formulae above to find the density of active sensors needed to obtain $|\mathcal{A}| I \sim 1$ when $|\mathcal{A}|$ is large:

$$
\begin{array}{ll}
\text { OPT } & p \rho=\frac{-p}{\log (1-p)} \frac{2}{\sqrt{27}} \log |\mathcal{A}|+o(\log |\mathcal{A}|) \\
\text { FLAT } & p \rho=\frac{-p}{\log (1-p)} \frac{1}{\pi} \log |\mathcal{A}|+o(\log |\mathcal{A}|) \\
\text { RAND } & p \rho=\frac{1}{\pi} \log |\mathcal{A}|+o(\log |\mathcal{A}|)
\end{array}
$$

Unlike the one dimensional case, the dominant term in the OP T and FLAT cases is different. The reason is that strategy FLAT needs about $\pi \rho$ sensors to fail in a small region because the minimum number of sensor failures for a hole occurs when all the sensors in a roughly circular region of radius 1 . However strategy OPT only needs about $(\sqrt{27} / 2) \rho$ sensors to fail. The ratio $(2 \pi / \sqrt{27}) \approx 1.209$ is just the density factor we lose in $\mathrm{OPT}$ as a result of the fact that disks do not tile the plane.

Strategy FLAT also has a better dominant term than strategy RAND, so at first sight it seems strategy FLAT should always win. In the limit as $I \rightarrow 0(|\mathcal{A}| \rightarrow \infty)$ this is indeed the case, but the simulation results in Figure 2 appear to show strategy $\mathrm{OPT}$ as better for a nontrivial range of values of $p$ and $\sigma$. To explain this discrepancy, we consider more carefully the expressions for $I_{\mathrm{OPT}}$ and $I_{\mathrm{FLAT}}$ above. Roughly speaking

$$
\begin{gathered}
I_{\mathrm{OPT}} \approx(2 / \sqrt{27})(1-p)^{(\sqrt{27} / 2) \rho}, \quad \text { while } \\
I_{\mathrm{FLAT}} \approx(p \rho)^{2}(1-p)^{\pi \rho} .
\end{gathered}
$$

The ratio is

$$
I_{\mathrm{FLAT}} / I_{\mathrm{OPT}} \approx 2.6(p \rho)^{2}(1-p)^{.5435 \rho} \approx 2.6(p \rho)^{2} e^{-.5435 p \rho} .
$$

Thus we see a significant polynomial factor and a small negative exponential factor. For modest target hole densities, strategy OPT can indeed beat strategy FLAT for small values of $p$ and $\sigma$. However for larger values (e.g., $p \rho \geq 11$ is enough), strategy FLAT is better.

\section{FUTURE WORK}

Although we provide a comprehensive comparison of various deployments here, several problems still remain open. First, derivation of density estimate for deterministic deployments in the presence of both failures and placement errors remains open. Our estimate for deterministic deployments is reliable only for zero placement error cases. Second, we only consider 1-full coverage here. Extension to the case of $k$-full coverage remains open. Third, we considered only the full coverage model here. A comparison of similar deployment strategies for other coverage models such as barrier coverage [6] and Trap Coverage [4] remain open.

\section{ACKNOWLEDGMENT}

This work was sponsored partly by NSF Grants CNS0721983, CCF-0728928, NIH Grant U01DA023812 from National Institute for Drug Abuse (NIDA), and FIT at the University of Memphis. The content is solely the responsibility of the authors and does not necessarily represent the official views of the sponsors.

\section{REFERENCES}

[1] R. Arratia, L. Goldstein, and L. Gordon, "Two Moments Suffice for Poisson Approximations: The Chen-Stein Method," Annals of Probability, vol. 17, pp. 9-25, 1989.

[2] X. Bai, S. Kumar, D. Xuan, Z. Yun, and T. H. Lai, "Deploying Wireless Sensors to Achieve Both Coverage and Connectivity," in ACM MobiHoc, 2006.

[3] P. Balister and S. Kumar, "Random vs. Deterministic Deployment of Sensors in the Presence of Failures and Placement Errors," University of Memphis, CS-09-002, Tech. Rep., 2008.

[4] P. Balister, Z. Zheng, S. Kumar, and P. Sinha, "Trap Coverage: Allowing Holes of Bounded Diameter in Wireless Sensor Networks," in IEEE INFOCOM, 2009.

[5] S. Bapat, V. Kulathumani, and A. Arora, "Analyzing the Yield of ExScal, a Large Scale Wireless Sensor Network Experiment," in IEEE ICNP, Boston, MA, 2005.

[6] S. Kumar, T. H. Lai, and A. Arora, "Barrier Coverage with Wireless Sensors," in ACM MobiCom, 2005.

[7] S. Kumar, T. H. Lai, and J. Balogh, "On $k$-Coverage in a Mostly Sleeping Sensor Network," in ACM MobiCom, 2004.

[8] S. Shakkottai, R. Srikant, and N. B. Shroff, "Unreliable Sensor Grids: Coverage, Connectivity and Diameter," in IEEE INFOCOM, vol. 2, San Francisco, CA, 2003, pp. 1073-1083.

[9] P. Wan and C. Yi, "Coverage by Randomly Deployed Wireless Sensor Networks," IEEE/ACM Transactions on Networking (TON), vol. 14, pp. 2658-2669, 2006.

[10] H. Zhang and J. Hou, "On Deriving the Upper Bound of $\alpha$-Lifetime for Large Sensor Networks," in ACM MobiHoc, 2004.

[11] - "Is Deterministic Deployment Worse than Random Deployment for Wireless Sensor Networks?" in IEEE INFOCOM, 2006. 